\title{
The Volatile and Heterogenous Gut Microbiota Shifts of COVID-19 Patients Over The Course of A Probiotics-Assisted Therapy
}

\section{Chunyan Wu}

Tongji University Tenth People's Hospital: Shanghai Tenth People's Hospital https://orcid.org/00000003-3192-3126

\section{Qian Xu}

Tongji University Tenth People's Hospital: Shanghai Tenth People's Hospital

\section{Zhan Cao}

Tongji University Tenth People's Hospital: Shanghai Tenth People's Hospital

\section{Dengdeng Pan}

Tongji University Tenth People's Hospital: Shanghai Tenth People's Hospital

\section{Ying Zhu}

Tongji University Tenth People's Hospital: Shanghai Tenth People's Hospital

\section{Sheng Wang}

Tongji University Tenth People's Hospital: Shanghai Tenth People's Hospital

\section{Danping Liu}

Shanghai Public Health Clinical Center

\section{Zhigang Song}

Shanghai Public Health Clinical Center

\section{Wei Jiang}

Tongji University Tenth People's Hospital: Shanghai Tenth People's Hospital

\section{Yumeng Ruan}

Reabio Genomics Institute

\section{Nan Qin ( $D$ qinnan001@126.com )}

Tongji University Tenth People's Hospital: Shanghai Tenth People's Hospital https://orcid.org/00000002-9128-9414

\section{Hongzhou Lu}

Shanghai Public Health Clinical Center

\section{Huanlong Qin}

Tongji University Tenth People's Hospital: Shanghai Tenth People's Hospital

\section{Research}


Keywords: COVID-19, microbiota, 16S rRNA gene sequencing, metatranscriptome, probiotics, therapy Posted Date: September 10th, 2020

DOI: https://doi.org/10.21203/rs.3.rs-72753/v1

License: (c) (1) This work is licensed under a Creative Commons Attribution 4.0 International License. Read Full License 


\section{Abstract}

Background Coronavirus disease 2019 (COVID-19) has rapidly become a global pandemic, and little is known regarding the gut microbiota dynamics of the disease that often features a drastic and swift progression. Here we employed analyses of 16S rRNA gene sequencing and metatranscriptome to investigate the gut microbiome characteristics of a group of COVID-19 patients over the course of a probiotics-assisted therapy.

Results The COVID-19 patients exhibited apparent microbiota alterations characterized by prominent compositional and functional shifts, which included taxonomic changes (e.g., increased relative abundance of Enterococcus and Rhodococcus, and decreased relative abundance of Faecalibacterium and Clostridium XIVa) and transcriptional changes (e.g., increased transcriptional activities of Escherichia coli and Klebsiella pneumoniae, virulence factors, and antibiotic resistance genes, and decreased activities of Faecalibacterium prausnitzii). Importantly, there were great interpersonal heterogeneity and intertimepoint fluctuations, as the most abundant or transcriptionally active taxa often greatly differed among individual patients and timepoints. Coincided with the resolution of respiratory symptoms, after the therapy some patients showed signs of recovery in the gut microbiome abnormalities. Associations were identified between gut and airway taxa and serum factors.

Conclusion Our findings suggested that there is a lack of gut microbiota stability in COVID-19 patients and that measures are needed to ameliorate the gut microbiome perturbations in the patients to improve the prognosis. In addition, inclusion of probiotics is safe for treating COVID-19 patients and may improve their prognosis.

Trial registration ISRCTN, ChiCTR2000029999. Registered 19 February 2020, http://www.chictr.org.cn/showprojen.aspx?proj=49717

\section{Backgroud}

Coronavirus disease 2019 (COVID-19) is a newly emerged infectious respiratory disease of probable zoonotic origin that has rapidly become a global pandemic. The causative agent of COVID-19 is severe acute respiratory syndrome coronavirus 2 (SARS-CoV-2), which requires the binding of viral spike proteins to host angiotensin-converting enzyme 2 (ACE2) receptors to initiate infection ${ }^{1}$. It has been reported that a considerable proportion (i.e., $3 \%-50 \%$ ) of COVID-19 patients had gastrointestinal symptoms (e.g., diarrhea, nausea, and vomiting) 234 , suggesting alterations in the gut environment. Recent reports showed substantial gut microbiota shifts in patients with SARS-CoV-2 ${ }^{567}$. However, because the disease progression is typically swift and drastic once the symptoms arise ${ }^{8}$, it is crucial to examine the microbial dynamics during the illness and convalescence phases.

Currently there is an urgent need to develop an effective cure for COVID-19, as world-wide vaccination may take years to accomplish and the treatment outcome remains unsatisfactory in many countries 9,10 . 
Given that the patients may manifest severe gastrointestinal symptoms and microbiota abnormalities, inclusion of probiotics into the medication may improve their treatment outcome. Here we employed a probiotics-assisted therapy to treat a group of COVID-19 patients, and the dynamics of gut microbiota and clinical manifestations was monitored over the course of treatment. We showed that the COVID-19 patients exhibited prominent gut microbiota shifts that were characterized by decreased microbial diversity, extensive taxonomic and transcriptional alterations, and great interpersonal heterogeneity and intertimepoint fluctuations. Our findings suggested a lack of relative stability in the gut microbiota of COVID-19 patients, which may help formulate strategies to improve the treatment outcome and posttreatment rehabilitation.

\section{Results}

\section{Treatment outcome of the patients with COVID-19}

We recruited 13 COVID-19 patients, 15 non-COVID-19 pneumonia controls, and 15 healthy controls. The median ages of the three groups were 66, 51, and 64 years old, respectively (Table S1). All COVID-19 patients displayed respiratory symptoms (Table S1) and several of them had gastrointestinal manifestations, including one individual with diarrhea. Two patients (COV06 and COV13) showed positive results for SARS-CoV-2 in stool based on viral RNA metagenomics.

The COVID-19 patients were treated with a probiotics-assisted therapy (see Materials and Methods). All patients were discharged after their COVID-19 tests turned negative. We monitored the alterations of some relevant clinical variables in 8 COVID-19 patients before and after the treatment that registered a minimal of 2-fold change (Table S2). Decreases of inflammatory indicators were common in the COVID19 patients after the treatment, which included C-reactive protein (CRP; 3 patients), tumor necrosis factor (TNF)-a (7 patients), interleukin-1 $\beta$ (7 patients), interleukin-2 (3 patients), interleukin-4 (7 patients), interleukin-5 (6 patients), interleukin-6 (5 patients), interleukin-8 (3 patients), interleukin-12P70 (7 patients), interferon (IFN)-a (3 patients), and interferon IFN- $\gamma$ ( 5 patients). The hematologic trait of white blood cell (WBC) was reduced in COV03 and COV12, whereas lymphocyte was elevated in COV06. In addition, alanine aminotransferase (ALT), lactate dehydrogenase (LDH), total bilirubin, and blood urea nitrogen were reduced in COV09, COV03, COV05, and COV05, respectively. These results suggested the overall reduced inflammation in the COVID-19 patients after the treatment.

\section{Taxonomic changes of gut microbiota in the patients with COVID-19}

Analysis of $16 \mathrm{~S}$ rRNA gene sequencing revealed that in comparison with the healthy controls and nonCOVID-19 pneumonia participants, the COVID-19 patients exhibited a reduction in microbial diversity (Shannon index, $P<0.05$ ) and a difference in microbial structure (ANOSIM, $P<0.05$, Fig. 1a). These alterations were accompanied by substantial taxonomic shift. Compared with the healthy controls, the COVID-19 patients exhibited the reduced relative abundances of Firmicutes at the phylum level and 21 genera including Faecalibacterium, Roseburia, Gemmiger, and Clostridium XIVa as well as increased proportions of Enterococcus, Lactobacillus, Rhodococcus, and Acinetobacter (Wilcoxon rank-sum test, 
FDR < 0.05; Table S4). Furthermore, there was prominent compositional heterogeneity in the COVID-19 patients, as the most dominant genera that often accounted for over half of the total abundance differed among the individuals. According to the most abundant genera at T0 (i.e., before the treatment), the COVID-19 patients were stratified into 5 clusters, with each being dominant with one or two genera (Fig. 1b). Specifically, the clusters 1 (COV02 and COV11), 2 (COV12), 3 (COV03, COV04, and COV10), 4 (COV07), and 5 (COV05, COV06, COV08, COV09, and COV13) were characterized by a high relative abundance (26\%-97\%) of Akkermansia (54\%-61\%) and Bacteroides (7\%-29\%); Lactobacillus (66\%) and Bifidobacterium (20\%); Bacteroides (59\%-87\%) and Alistipes (27\%) or Enterococcus (11\%); Enterococcus (97\%); Escherichia/Shigella (26\%-66\%) and multiple taxa including Anaerostipes (48\%-16\%), Enterococcus (17\%), Klebsiella (24\%), Akkermansia (18\%) or Phascolarctobacterium (33\%). Of note, two patients (i.e., COV06, COV13) with detectable SARS-CoV-2 RNA in stool were dominated with Escherichia/Shigella. We also detected microbial differences in the upper respiratory tract between the non-COVID-19 controls and COVID-19 patients and that there were likewise apparent interpersonal variations among individual COVID-19 patients. For example, the dominant genera were Prevotella and Veillonella in the pneumonia controls and Staphylococcus (COV02), Enterococcus (COV03, COV04, COV06, COV07), Staphylococcus, Pseudomonas (COV05), Streptococccus (COV08), Ralstonia (COV09), Rothia (COV10), Prevotella (COV11), and Neisseria (COV12) in the COVID-19 patients, respectively (Fig. S1).

Analysis of the microbial transcriptional activity revealed that compared with the healthy controls, the COVID-19 patients were characterized by augmented presence of 322 species, including Escherichia coli, Lactobacillus delbrueckii,Salmonella enterica, Providencia alcalifaciens, Staphylococcus auricularis, Klebsiella pneumoniae, and Enterococcus faecium, and decreased presence of 279 species, including Bacteroides vulgatus, Faecalibacterium prausnitzii, Bacteroides cellulosilyticus, Bacteroides dorei, and Eubacterium eligens (Wilcoxon rank-sum test, FDR < 0.05; Fig. 1c and Table S3). There was some noticeable coincidence between the taxonomic and transcriptional shifts, as evidenced by the genera or affiliating species of Faecalibacterium, Enterococcus, Rhodococcus, Acinetobacter, Roseburia, Blautia,Parabacteroides, Dialister, and Ruminococcus. As such, the transcriptomics-based analysis further underscored the substantial changes in gut microbial community in the COVID-19 patients.

We next examined the strain-level variations in the COVID-19 patients. To this end, we applied StrainPhIAn to build the phylogenetic trees based on single nucleotide variants (SNVs) in species-specific marker genes, which revealed considerable strain-level heterogeneity of Escherichia coli in the patients with COVID-19 (Fig. S2). Specifically, the dominant strains were phylogenetically close to E. coli UMN026 in patient COV11, to E. coliUMEA_3212-1 in patient COV13, and to E. coli KTE196 in patients COV01, COV06, COV09 and COV12, respectively. Hence, our analyses revealed that COVID-19 was associated with taxonomic and transcriptional shifts in the gut and/or airway microbiotas and that there was great heterogeneity among individual patients.

\section{Functional shift of gut microbiota in the patients with COVID-19}


Having revealed the clear taxonomic alterations of gut microbiota in the COVID-19 patients, we next sought to examine the microbial functional characteristics using the transcriptomic data. Of the 5,125 genes functionally annotated to Kyoto Encyclopedia of Genes and Genomes (KEGG) orthologous groups (KOs), 1,982 exhibited differential transcriptional activities between the HCs and COVID-19 patients, including 866 decreased and 1,116 increased in COVID-19. At pathway level, the COVID-19 patients were characterized by enrichment of 11 MetaCyc pathways including beta-Lactam resistance, Biofilm formation-Escherichia coli, and Bacterial invasion of epithelial cells as well as depletion of 86 MetaCyc pathways, most of which belonged to the categories of amino acid metabolism, lipid metabolism, and carbohydrate metabolism (Table S5). In addition, the expression level of several virulence factors in the COVID-19 patients was significantly higher than both the healthy controls and pneumonia controls (Fig. 1d). The top 5 transcriptionally active virulence factors in the COVID-19 patients were Enterobactin (VF0228), ECP (VF0404), T2SS (VF0333), Type 1 fimbriae (VF0221), and Yersiniabactin (VF0136), collectively accounting for $61.9 \%$ of the total transcriptomic abundance (Fig. 1e, Table S6). In addition, the top 5 contributing species of virulence factors in the COVID-19 patients were Escherichia coli, Shigella dysenteries, Yersinia pestis, Yersinia enterocolitica, and Salmonella enterica, collectively accounting for $98.8 \%$ of the total transcriptomic abundance (Fig. 1f, Table S7). The prominent presence of virulence factors and their contributing pathogens or pathobionts illustrated the deteriorating gut flora in the COVID-19 patients.

We next investigated the profile of antibiotic resistance genes in gut microbiota of the participants. Genes potentially conferring resistance to 35 different antibiotics were identified in the COVID-19 patients, of which Macrolide, Tetracycline, Penam, Diaminopyrimidine, and Phenicol were the most transcriptionally active (Fig. S3a, Table S8). The expression of antibiotic resistance genes (ARGs) was significantly increased in COVID-19 (Fig. 1d), with the most abundant types being resistance-nodulation-cell division (RND) antibiotic efflux pump, Erm 23S ribosomal RNA methyltransferase, major facilitator superfamily (MFS) antibiotic efflux pump, tetracycline-resistant ribosomal protection protein, and pmr phosphoethanolamine transferase (Fig. S3b, Table S9). Similar to our observation in taxonomic analyses (Fig. 1b and Fig. S1), there were great interpersonal differences in ARGs. For example, the most transcriptionally active ARGs were Erm 23S ribosomal RNA methyltransferase in COV04, COV10, and COV11, RND antibiotic efflux pump in COV05, COV06, COV07, COV08, COV09, COV12, and COV13, tetracycline-resistant ribosomal protection protein in COV01, COV03, and COV13, and CfXA betalactamase in COV02, respectively. Overall, our findings revealed extensive alterations of gut microbiota in COVID-19 in both taxonomic and functional aspects, which featured prominent variations among individual patients.

\section{Association between gut microbiome and disease severity of COVID-19}

We next investigate the correlation of gut or upper airway microbes with a set of clinical indicators (Fig. 2a). Among upper airway taxa, Bacteroides, Akkermansia, and Enterococcus showed negative association with CD3 and CD4. Among gut taxa, Rhodococcus and Acinetobacter showed negative association with CD3, CD4, CD45, haemoglobin $(\mathrm{Hb})$ concentrations, and red blood cells (RBC); 
Bacteroides and Veillonella showed positive association with haemoglobins, RBC, and CD3, respectively; Enterococcus showed positive association with plasma concentration of carbon dioxide.

We also detected significant correlations between microbial functional components and clinical indicators. The expression level of bacteria virulence factors (e.g., Yersiniabactin, Salmochelin, Shu, and SgrA) were negatively correlated with CD8 (Fig. 2b), whereas multiple antibiotic resistance genes (e.g.,beta lactamase, chloramphenicol acetyltransferase (CAT), undecaprenyl pyrophosphate related proteins, and multidrug and toxic compound extrusion (MATE) transporter) were negatively associated with CD3 (Fig. 2c). These findings indicated that gut and airway microbiota shifts were linked to the clinical manifestations of inflammation, possibly related to COVID-19.

\section{Convalescence coincided with the recovery of dysbiosis in the COVID-19 patients}

To investigate the dynamics of the gut microbiome in the COVID-19 patients during the process of treatment, we compared the taxonomic data among the baseline ( $\mathrm{TO}$, first sampling date prior to the probiotics-assisted therapy), 7-day posttreatment (T1), and 14-day posttreatment (T2) for each patient. Among the 12 recruited COVID-19 patients, stool samples both before and after the therapy could only be collected from 8 individuals (Fig. 1b). At the end of the treatment, the 8 COVID-19 patients showed a partial rise in the microbial diversity (Wilcoxon rank-sum test, $P<0.05$ ) and 6 out of the 8 COVID-19 patients (75\%) showed a partial microbial compositional "recovery", evidenced by a decrease of BC (BrayCurtis) distance to the healthy group (Fig. 3a). This alteration was accompanied by substantial taxonomic shift, such as COVID-19-increase genera Enterococcus and Rhodococcus registered at least 2fold reduction in 3 patients (COV03, COV05, and COV12) and 4 patients (COV05, COV08, COV09, and COV11), respectively, and COVID-19-depleted genera Faecalibacterium and Roseburia exhibited at least 2fold increase in 3 patients (COV11, and COV12) and 4 patients (COV03, COV06, COV09, and COV12), respectively (Table S10). This partial compositional "recovery" was accompanied by a similar trend in the microbial transcriptome. After treatment, $23 \%-63 \%$ of the species that had COVID-19-associated transcriptional elevation exhibited at least 2 -fold reduction, whereas $7 \%-67 \%$ of the species that had COVID-19-associated transcriptional reduction exhibited at least 2-fold increase (Table S11). For example, the major gut commensal bacterium Faecalibacterium prausnitzii was increased in patients COV05, COV08, COV09, COV11, and COV12; butyrate-producing anaerobic bacterium Roseburia hominis was increased in patient COV05, COV09, COV11, and COV12. On the contrary, opportunistic pathogen Escherichia coli was decreased in all patients except COV03; Salmonella enterica was decreased in COV05, COV06, COV08, COV09, and COV12; Providencia alcalifaciens was decreased in all patients except COV03 and COV11; Staphylococcus auricularis was decreased in COV03, COV07, COV11, and COV12; Klebsiella pneumoniae was decreased in all patients except COV03 and COV05 (Fig. 3b). The findings suggested that the treatment correlated with a noticeable recovery of the gut microbiota perturbations in the COVID-19 patients.

An important finding of the longitudinal $16 \mathrm{~S}$ data was that there were great intertimepoint compositional variations of gut microbiome in most COVID-19 patients (Fig. 1b). In patients COV03, COV05, COV06, and 
COV08, the most dominant genus at T0 exhibited a great reduction in relative abundance at the end of treatment, whereas the most dominant genus at the end of treatment was barely detectable at T0. For example, the top taxa of patient COV03 were Bacteroides and Lactobacillus before and after the treatment, respectively; the top taxa of patient COV05, COV06, and COV08 was Escherichia/Shigella prior to the treatment, as were Bacteroides, Enterococcus, and Veillonella at the end of treatment. The prominent microbiota changes were also evident in patient COV11, whose shares of Akkermansia and Bacteroides were $54 \%$ and $29 \%, 75 \%$ and $5 \%$ or $19 \%$ and $44 \%$ at T0, T1 or T2. For the airway microbes, the drastic intertimepoint changes were present in only 3 patients. Specifically, the top genera of patients COV01, COV03, and COV03 were Ralstonia, Clostridium sensu stricto, and Pseudomonas at T0 and Lactobacillus, Lactobacillus, and Corynebacterium at T2, respectively (Fig. S1).

The drastic compositional shift over the course of treatment was mirrored by the bacterial transcriptinal activities. In patients COV03, COV05, COV06, COV09, and COV12, the most transcriptionally active species differed between the first and last timepoints. For example, the top species of patient COV03 were Bacteroides fragilis before the treatment and Yersinia enterocolitica afterwards. Of note, there was apparent divergence between the most abundant taxa and most transcriptionally active microbes. For example, in patient COV01, the most abundant taxon was Enterococcus, whereas the most transcriptionally active species was Bacillus cereus; in patient COV12, Fecaelibacterium prausnitzii was transcriptionally active, although the genus was barely detected in 16S rRNA gene-based analysis. However, noticeable coincidence can be found for Escherichia/Shigella and E. coli in patients COV05, COV06, and COV09, Akkermansia and A. muciniphila in patient COV11, Klebsiella and K. pneumoniae in patient COV06, and Veillonella and V. parvula in patient COV08. Overall, our longitudinal analyses revealed that over the course of treatment, the COVID-19 patients exhibited apparent recovery in the disease-associated microbial abnormalities and that the gut microbiota of the COVID-19 patients showed drastic intertimepoint variations.

\section{Discussion}

In this study, we characterized the dynamic composition and transcriptomics of gut microbiota in patients with COVID-19 over the course of treatment. The patients with COVID-19 showed prominent gut microbiome shifts from healthy controls and non-COVID-19 that were characterized by taxonomic and transcriptional differences as well as great interpersonal heterogeneity and intertimepoint variations. This was accompanied by microbial alterations in the upper airway. The taxonomic shifts included decreased relative abundances of Faecalibacterium, Roseburia, and Clostridium XIVa as well as increased proportions of Enterococcus, Rhodococcus, and Acinetobacter. The transcriptional alterations in the gut microbiota included the augmented presence of opportunistic pathogens or pathobionts (e.g., Escherichia coli, Salmonella enterica, Staphylococcus auricularis, and Klebsiella pneumoniae), virulence factors, and ARGs as well as diminished presence of Faecalibacterium prausnitzii, a major gut commensal. These changes indicated the deteriorating gut ecosystem in the patients. In addition, correlations were detected between microbial components (e.g., gut microbes, bacteria virulence factors, antibiotic resistance genes, and airway microbes) and inflammatory indicators (e.g., CD3, CD4, haemoglobin concentrations, and red 
blood cells). After treatment, the COVID-19 patients exhibited a partial recovery of the gut microbiome perturbations, evidenced by the increased microbial diversity as well as a partial microbiota recovery: that is the "approaching" of the COVID-19-associated taxonomic and transcriptional profiles to those in healthy controls. Hence, our findings illustrated the complex changes occurring in the gut and upper airway microbial communities of COVID-19 patients that are pivotal to understand the disease and its therapy.

Intestinal flora is essential for the maintenance of host immunity and homeostasis. The microbial community may regulate host immune and inflammatory responses along the gut-lung axis via microbial metabolites and the mucosal immune system, thereby playing a pivotal role in host health ${ }^{11}$. An important feature of the human gut microbiota is its relative stability, which may be sustained for one year ${ }^{12}$ or even decades ${ }^{13}$. In contrast, the COVID-19 patients exhibited great intertimepoint fluctuation. Such fragile, unstable gut microbiota in COVID-19 patients is likely attributed to the reduced microbial diversity as well as decreased presence of some major commensals (e.g., Faecalibacterium prausnitzii). In light of this, measures targeting the COVID-19-associated microbiota abnormalities could be a crucial avenue of multi-pronged therapies.

In severe COVID-19 cases, the infection can trigger a series of immune responses or cytokine storms that correlate with many of the symptoms. In our study, the probiotics-assisted therapy led to clinical improvements in COVID-19 patients, which was characterized by the reduced inflammation (e.g., tumor necrosis factor (TNF)-a, interleukin-1 $\beta$, interleukin-4, and interleukin-12P70). It worth noting that a high dosage of probiotics (i.e., ca. 200 billion colony forming units of bacterial cells daily) was prescribed for the COVID-19 patients, who showed no adverse reactions (e.g., diarrhea) linked to the auxiliary therapeutics. The results suggested that the probiotics supplementation is safe and is amenable for inclusion into other medications for COVID-19 patients who may have gut microbiota abnormalities.

\section{Conclusions}

We identified substantial COVID-19-associated gut and upper airway microbiota shifts, including alterations in taxonomic composition and transcriptional activities. In addition to resolving the respiratory symptoms in COVID-19 patients, a probiotics-assisted therapy correlated with partial recovery of the microbiota perturbations. These findings provided new insights into the gut and airway microbiome characteristics of COVID-19 that may have crucial clinical implications.

\section{Materials And Methods}

\section{Recruitment of participants}

This study recruited 13 hospitalized COVID-19 patients, 15 hospitalized patients with community acquired pneumonia (pneumonia controls) and 15 healthy healthy controls (Table S1). SARS-CoV-2 infection was detected by dual RT-PCR test targeting two different regions of the RdRp gene. Non-COVID- 
19 pneumonia controls were patients admitted with community-acquired pneumonia who were tested negative for SARS-CoV-2. Healthy controls were individuals with no past medical history or history of antibiotic intake in the past 3 months and tested negative for SARS-CoV-2. All participants were admitted to Shanghai Public Health Center (SHPHC, Shanghai, China). Written informed consent was obtained from all participants or their families. All procedures were performed in compliance with the Declaration of Helsinki.

Epidemiological, clinical, radiological characteristics, laboratory, and treatment data were obtained from the electronic medical records. Clinical information included demographic data, medical history, exposure history, underlying comorbidities, symptoms, signs, laboratory findings, chest computed tomographic (CT) scans, and treatment measures (ie, antiviral therapy, antibiotics therapy, respiratory support).

\section{Probiotics-assisted therapy}

Each COVID-19 and Non-COVID-19 pneumonia patient was prescribed with a cocktail of antiviral drugs, antibiotics, thymosin, vitamin $\mathrm{C}$, and Ulinastatin and 4 tablets of probiotics daily. Each probiotics tablet contains at least 50 billion colony forming units of bacterial cells comprising the following strains: $B$. lactis subsp. HNO19, L. casei subsp. Lc-11, L. plantarum subsp. Lp-15, B. lactis subsp. B420, B. longum subsp. BL05, L. format subsp. Lg-36, L. rhamnosus subsp. Lr-32, L. paracasei subsp. Lpc-37, and L. salivarius.

\section{DNA and RNA extraction}

Fresh fecal samples were collected from all enrolled individuals and then stored at $-80^{\circ} \mathrm{C}$ within two hours. DNA was extracted from frozen fecal samples using QIAamp Fast DNA Stool Mini Kit (Qiagen, Hilden, Germany) according to the manufacturer's instructions. The concentration of DNA was measured by NanoDrop (Thermofisher, USA). Viral RNA from stool samples was extracted using QIAamp Viral RNA Mini Kit (Qiagen, Hilden, Germany).

\section{$16 S$ rRNA gene sequencing analysis}

DNA from samples was amplified using primers targeting the V3-V4 regions of 16S rRNA gene (341F and 806R). Amplicon libraries were sequenced on Illumina Miseq platform for paired end reads of 300bp. High quality reads were abstained after as below (average quality score $>=20$; ambiguous $\mathrm{N}$ base $<=3$ ). Paired-end reads were merged by pandaseq ${ }^{14}$ with overlap. Merged sequences were clustered to generate operational taxonomic units (OTUs) clustering at the $97 \%$ similarity level after chimeras filtering, using UPARSE ${ }^{15}$. A representative sequence of each OTU was assigned to a taxonomic level in the RDP database ${ }^{16}$ using the RDP classifier at an $80 \%$ confidence level ${ }^{17}$.

\section{Metatranscriptomicanalysis}

No rRNA depletion steps were performed due to the limited biomass of the starting material. Doublestranded cDNA was synthesized with the QuantiTect Rev. Transcription Kit (Qiagen, Hilden, Germany) and 
NEBNext RNA Second Strand Synthesis Module Kit (New England BioLabs, Ipswich, Massachusetts, USA), and amplified with the Hieff NGS One Pot DNA Library Prep Kit for Illumina (Yeasen, Shanghai, China). The quality of all libraries was evaluated using an Agilent bioanalyser (Agilent Technologies, USA) with a DNA LabChip 1000 kit. Metatranscriptomic sequencing of samples was conducted on Illumina Hiseq 4000 platform with 150bp paired-end read length.

Metatranscriptomic reads were taxonomically profiled using Kraken ${ }^{18}$ with default parameter settings. HUMAnN2 ${ }^{19}$ was used to calculate the relative abundance of metabolic pathways in MetaCyc database. ShortBRED ${ }^{20}$ was used to quantify the abundance of antibiotic resistance genes and virulence genes against the CARD database ${ }^{21}$ and Virulence Factors Database ${ }^{22}$. All analyses were performed on gene abundances normalized to reads per kilobase per million reads (RPKM). Strain-level profiling was performed with StrainPhIAn ${ }^{23}$. For each sample, the clean reads were first mapped against the MetaPhIAn2 markers by Bowtie2 and then the consensus sequence was produced according to the mapping result. Finally, the extracted consensus sequences of references and samples were multiply aligned by MUSCLE ${ }^{24}$, and the phylogenetic trees were built by RAxML ${ }^{25}$ (parameters: -m GTRCAT and $-\mathrm{p}$ 1234).

\section{Statistics}

To detect significant differences in relative abundance of taxon features, the nonparametric Wilcoxon test (wilcox.test in R) was performed with false discovery rate (FDR) $<0.05$ (Benjamini-Hochberg), and the enrichment group was then determined according to the higher rank-sum.

\section{Declarations}

\section{Ethics approval and consent to participate}

This study was approved by the institutional review boards at Shanghai Public Health Center (SHPHC, Shanghai, China). Written informed consent was obtained from all participants or their families.

\section{Consent for publication}

Not applicable.

\section{Availability of data and material}

Sequencing dataset was deposited to the NCBI Sequence Read Archive under BioProject accession number PRJNA649161.

\section{Competing interests}

The authors declare that they have no competing interests. 


\section{Funding}

This work was supported by a specialized funding for Covid-19 from the 10th Hospital of Tongji University (04.02.20001).

\section{Authors' contributions}

H.L.Q. conceptualized and led the project. H.L.Q., N.Q., and H.Z.L designed the study. C.Y.W., Q.X., and Z.C. led the data analyses. Z.C. and Z.G.S. organized collection of samples and documentation of clinical measures. C.Y.W., Q.X., and Z.C. wrote the manuscript. D.D.P., Y.Z., S.W., D.P.L. and Z.G.S. assisted sample collection, documentation of clinical measures, and compilation of data. X.X. assisted data analyses. All authors read the manuscript and offered valuable suggestions.

\section{Acknowledgements}

Not applicable.

\section{References}

1. Zheng, Y.-Y., Ma, Y.-T., Zhang, J.-Y. \& Xie, X. COVID-19 and the cardiovascular system. Nature Reviews Cardiology17, 259-260 (2020).

2. Huang, C., et al. Clinical features of patients infected with 2019 novel coronavirus in Wuhan, China. The lancet395, 497-506 (2020).

3. Han, C., et al. Digestive symptoms in COVID-19 patients with mild disease severity: clinical presentation, stool viral RNA testing, and outcomes. The American journal of gastroenterology (2020).

4. Pan, L., et al. Clinical characteristics of COVID-19 patients with digestive symptoms in Hubei, China: a descriptive, cross-sectional, multicenter study. The American journal of gastroenterology115(2020).

5. Zuo, T., et al. Alterations in Gut Microbiota of Patients With COVID-19 During Time of Hospitalization. Gastroenterology0(2020).

6. Gu, S., et al. Alterations of the Gut Microbiota in Patients with COVID-19 or H1N1 Influenza. Clinical Infectious Diseases, ciaa709 (2020).

7. AKTAŞ, B. \& Aslim, B. Gut-lung axis and dysbiosis in COVID-19. Turkish Journal of Biology44, 265$272(2020)$.

8. Yuki, K., Fujiogi, M. \& Koutsogiannaki, S. COVID-19 pathophysiology: A review. Clinical Immunology215, 108427 (2020).

9. Wang, Y., et al. Remdesivir in adults with severe COVID-19: a randomised, double-blind, placebocontrolled, multicentre trial. The Lancet395, 1569-1578 (2020).

10. Women, T.P.J.J.B.A.T.A.T.A.L.P., Trust, C.a.T.H.H.N.F., Pharmacist, S.S.S.S.A.R.C., Pharmacist, B.A.B.A.A.C. \& Foundation, t.H.H.N. Investigational treatments for COVID-19. in Pharmaceutical 
Journal.

11. Marsland, B.J., Trompette, A. \& Gollwitzer, E.S. The gut-lung axis in respiratory disease. Annals of the American Thoracic Society12, S150-S156 (2015).

12. Dethlefsen, L. \& Relman, D.A. Incomplete recovery and individualized responses of the human distal gut microbiota to repeated antibiotic perturbation. Proceedings of the National Academy of Sciences108, 4554-4561 (2011).

13. Biagi, E., et al. Through ageing, and beyond: gut microbiota and inflammatory status in seniors and centenarians. PloS one5, e10667 (2010).

14. Masella, A.P., Bartram, A.K., Truszkowski, J.M., Brown, D.G. \& Neufeld, J.D. PANDAseq: paired-end assembler for illumina sequences. BMC bioinformatics13, 31 (2012).

15. Edgar, R.C. UPARSE: highly accurate OTU sequences from microbial amplicon reads. Nature methods10, 996-998 (2013).

16. Cole, J.R., et al. Ribosomal Database Project: data and tools for high throughput rRNA analysis. Nucleic acids research42, D633-D642 (2014).

17. Kuczynski, J., et al. Using QIIME to analyze 16S rRNA gene sequences from microbial communities. Current protocols in microbiology27, 1E. 5.1-1E. 5.20 (2012).

18. Wood, D.E. \& Salzberg, S.L. Kraken: ultrafast metagenomic sequence classification using exact alignments. Genome biology15, 1-12 (2014).

19. Franzosa, E.A., et al. Species-level functional profiling of metagenomes and metatranscriptomes. Nature methods15, 962-968 (2018).

20. Kaminski, J., et al. High-specificity targeted functional profiling in microbial communities with ShortBRED. PLoS computational biology11, e1004557 (2015).

21. Jia, B., et al. CARD 2017: expansion and model-centric curation of the comprehensive antibiotic resistance database. Nucleic acids research, gkw1004 (2016).

22. Chen, L., et al. VFDB: a reference database for bacterial virulence factors. Nucleic acids research33, D325-D328 (2005).

23. Truong, D.T., Tett, A., Pasolli, E., Huttenhower, C. \& Segata, N. Microbial strain-level population structure and genetic diversity from metagenomes. Genome research27, 626-638 (2017).

24. Edgar, R.C. MUSCLE: multiple sequence alignment with high accuracy and high throughput. Nucleic acids research32, 1792-1797 (2004).

25. Stamatakis, A. RAxML version 8: a tool for phylogenetic analysis and post-analysis of large phylogenies. Bioinformatics30, 1312-1313 (2014).

\section{Figures}


a
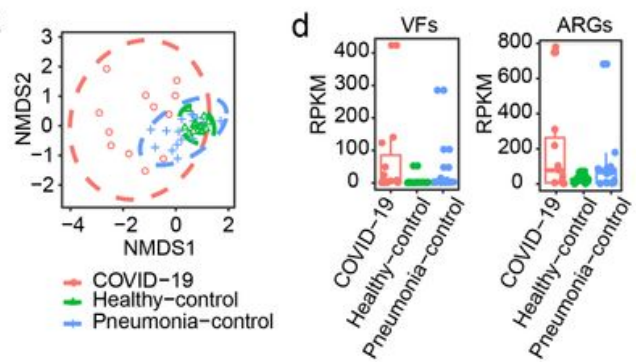

b

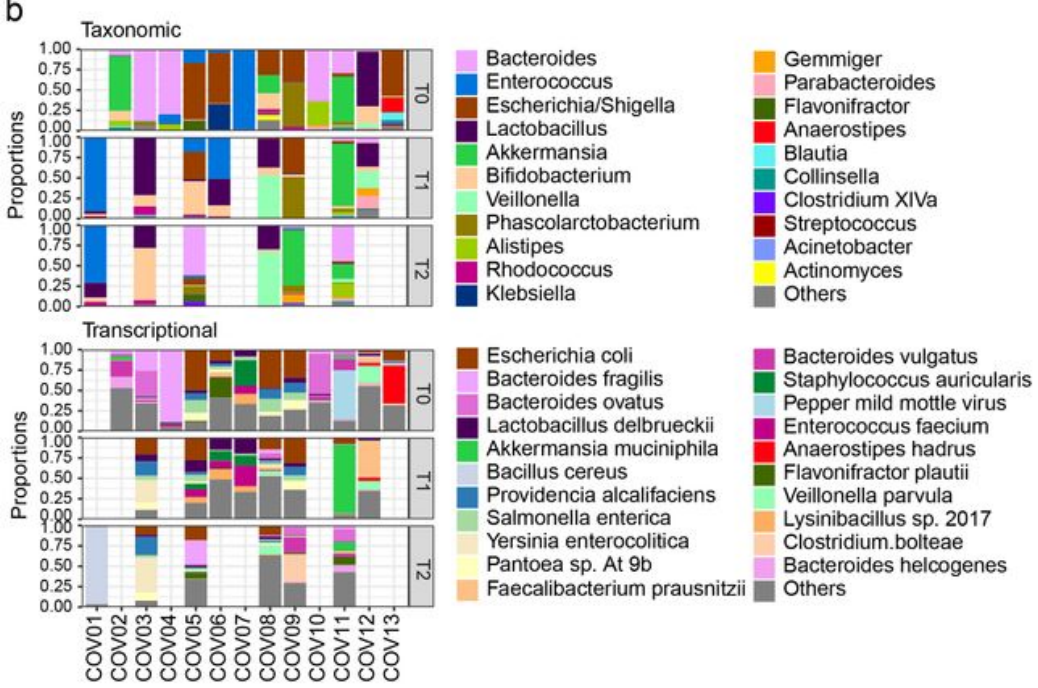

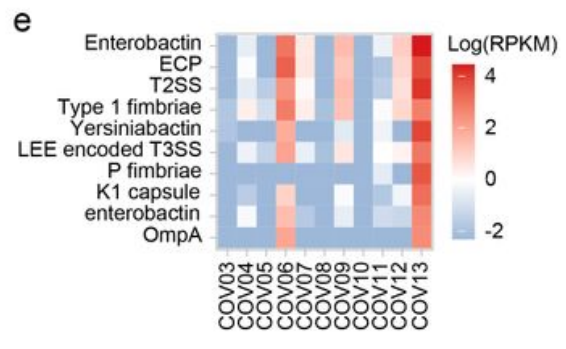

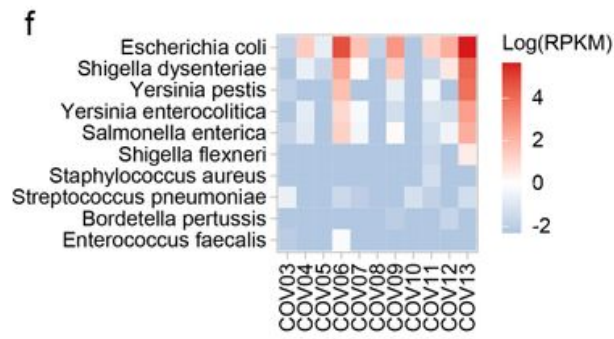

C

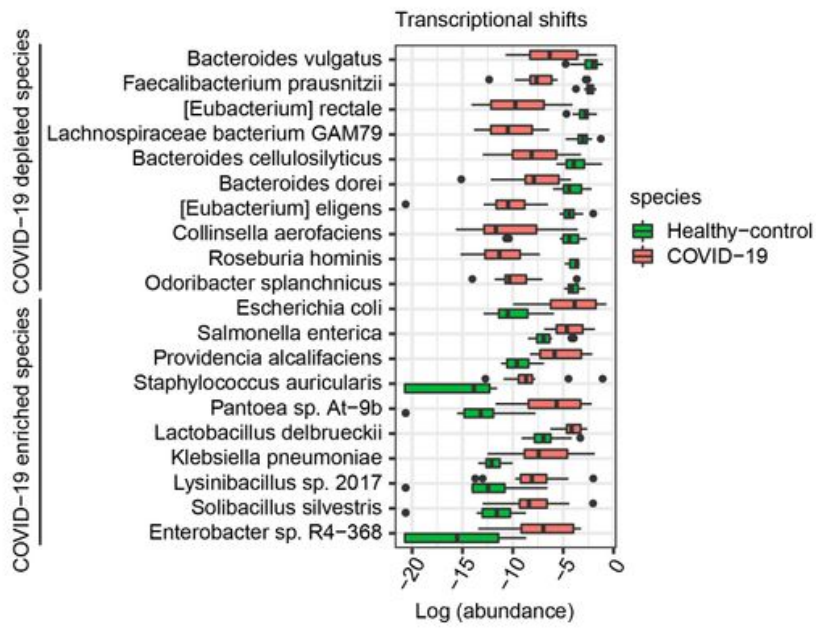

Figure 1

Shift of gut microbiota in patients with COVID-19. a. Microbial community alterations in COVID-19, viewed by NMDS (Non-metric multidimensional scaling) plot based upon Bray-Curtis dissimilarities. The microbial compositions were compared between healthy controls ( $n=15)$, COVID-19 ( $n=12)$, and pneumonia controls $(n=15)$. b. Relative abundance of top 20 genera and transcriptionally active species in COVID-19. c. Top 20 transcriptionally active species that were associated with COVID-19 (FDR < 0.05). d. Box plot showing the RPKM values of virulence factors (VFs) and antibiotic resistance genes (ARGs). e, $\mathrm{f}$. Box plots showing the top 10 most abundant virulence factors (e) and contributing species of virulence factor (f).
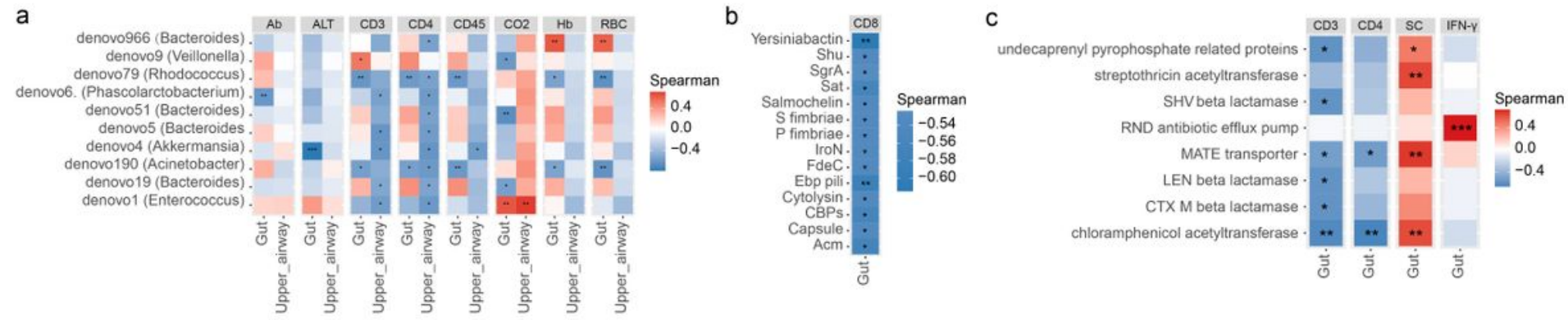

\section{Figure 2}

Correlation of features of gut microbiota with clinical indicators. a-c. Pairwise Spearman's correlation matrix of the OTUs (a), antibiotic resistance genes (b) and virulence factors (c) associated with different 


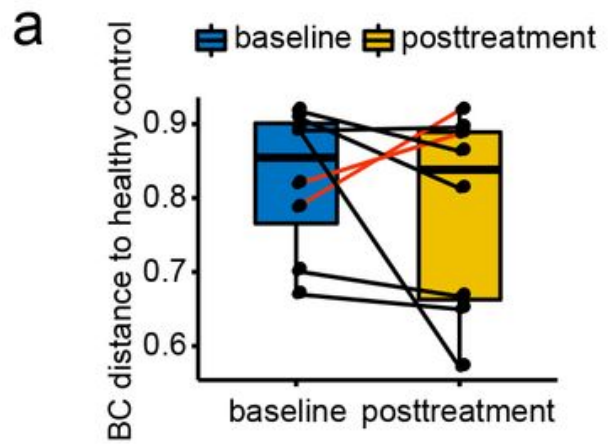

b

Alteration of microbial transcriptional activitiy posttreatment

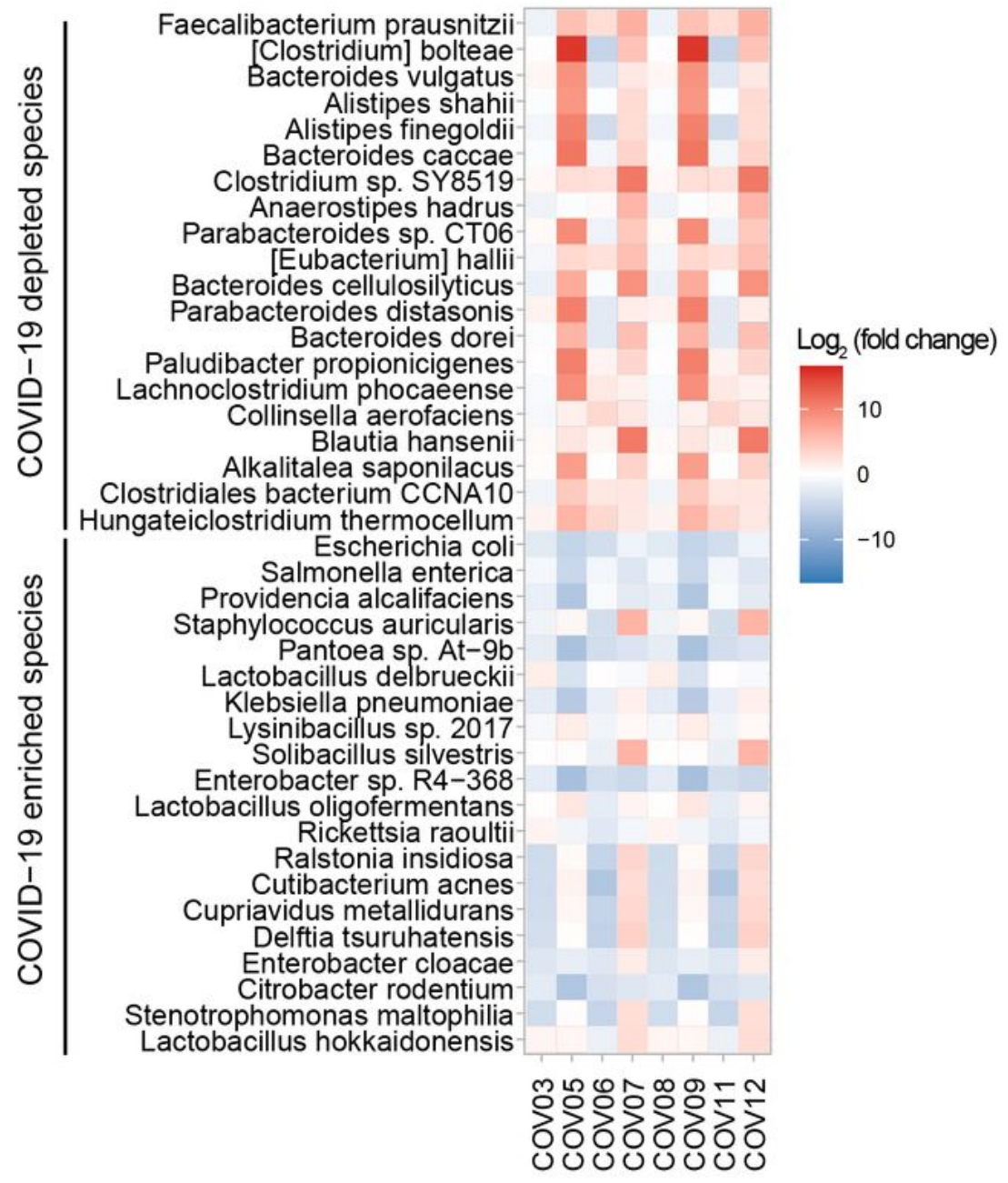

Figure 3

Shifts of gut microbiome in COVID-19 before and after the treatment. a. Dissimilarity of the gut microbiota of COVID-19 patients to that of healthy controls before and after treatment. The microbiota dissimilarity was calculated as bray-curtis dissimilarity. b. The alteration of COVID-19 enriched species (c) and depleted species of transcriptional activity after treatment. 


\section{Supplementary Files}

This is a list of supplementary files associated with this preprint. Click to download.

- Supplementaryinformation.docx

- Additionalfile2.docx

- Additionalfile1.xlsx 